\title{
Khuff Sequence 5 (KS5), Oman Mountains: Lateral Facies and Sequence Variability - a Record of Differential Subsidence?
}

\author{
L. Walz (University of Tuebingen, <liswalz@gmail.com>) and Thomas Aigner (University of \\ Tuebingen)
}

This study focuses on the sequence stratigraphy and 3-D modelling of the Khuff Sequence 5 (KS5) in the Oman Mountains (Al Jabal al-Akhdar), Sultanate of Oman. The Lower Khuff sequences (KS5 and KS6) are at this stage poorly understood, since most of the reservoir units are located in the Upper and Middle Khuff sequences (KS1 to KS4). Previous studies (Koehrer et al., 2010, 2011) revealed layer-cake geometries of grainstone bodies in the Middle and Upper Khuff sequences (KS1 to KS4) on outcrop to exploration scales. The key question of this study was if the layer-cake geometries continue to be present or are different in the KS5.

\section{Database and Workflow}

Five outcrop sections in the Oman Mountains form the basis for this project. The sections were logged sedimentologically on a 1:100 scale. In total $1,080 \mathrm{~m}$ of KS5 strata was investigated. Data on lithology, sedimentary structures and textures, facies types, components and rock colour were noted. For the determination of facies types an established Khuff facies atlas (Koehrer et al., 2010) was applied. A total of 227 thin sections were analysed for detailed interpretation of depositional environments. Furthermore the natural gammaradiation of the outcrop strata was measured with a spacing of every half a metre.

The concept of dynamic stratigraphy (Aigner, 1985; Kerans and Tinker, 1997; Aigner et al., 1998) was applied as workflow for this project. Based on the 1-D analysis of sequences and their stacking patterns, 2-dimensional correlations were constructed, that built the framework for the final 3-dimensional models.

\section{Sedimentological Analysis}

Based on the detailed sedimentological analysis and thin-section investigations 11 different lithofacies types could be identified in the KS5. These were grouped into six lithofacies associations, which were 3-dimensionally modelled: (1) tidal flat, (2) low-energy backshoal, (3) moderate-energy backshoal, (4) high-energy shoal, (5) moderate-energy fore-shoal, and (6) low-energy off-shoal. Since several facies types can either represent fore-shoal or backshoal deposits, it was crucial to find indicators that allow the differentiation between foreshoal and back-shoal. Several skeletal as well as non-skeletal particles were found to provide indicator criteria.

One-dimensional sequence stratigraphy: The 1-D sequence-stratigraphic analysis revealed several orders of cyclicity. The smallest scale of cycles was identified directly in the outcrops. A maximum of 66 small-scale cycles were recorded. Taking the 6.8 million years of KS5 into account the resulting average time span of a cycle comprises 103,000 years and can be assigned to the fifth-order Milankovitch cycles. The small-scale cycles could be grouped into genetically related medium-scale cycle sets. With a maximum of 21 medium-scale cycle sets an average cycle set duration of about 324,000 years results. They probably represent the fourth-order Milankovitch signals. Furthermore four high-frequency sequences (HFS) could be identified, marked by very characteristic gamma-ray patterns. It was possible to correlate these HFSs with subsurface well data. The whole Khuff Sequence KS5 represents one thirdorder composite sequence. 
Two-dimensional correlations: Two different correlation strategies were applied: the first one is based on the appearance of the four HFSs; the second one does not take the HFSs into account. Due to thickness variations between the different sections, lateral facies changes (Figure 1) as well as differential subsidence, complex correlation geometries, such as apparent downlaps, resulted. Thus, the KS5 differs markedly from the relatively simple layercake pattern of the Middle and Upper Khuff.

Three-dimensional models: Different 3-D models (based on the different correlation versions as well as different algorithms) clearly display the lateral facies changes, thickness differences and depositional geometries (Figure 2). The appearance of shoal facies, representing possible reservoirs, is governed by palaeogeography and stratigraphic position. The proximal sections in the northwest show a high percentage of shoal facies throughout the KS5 whereas in the more distal sections in the southeast the shoal facies appears mainly around sequence boundaries of the HFSs. Due to dynamic lateral facies changes throughout the KS5, the lateral extent of reservoir bodies is limited.

\section{Conclusions}

In contrast to the Middle and Upper Khuff sequences (KS1 to K4) the Khuff Sequence 5 shows marked facies and thickness variability, which makes sequence-stratigraphic correlations challenging. The overall layer-cake appearance of the Khuff sequences 1 to 4 is not observed in the KS5. There, probably due to proceeding rifting accompanied by differential subsidence a more complex pattern resulted. The overall high percentage of backshoal environment with intercalated grainy shoal intervals suggests that the KS5 could be a good facies analogue to the subsurface North Field and South Pars area.

\section{References}

Aigner, T. 1985. Storm depositional systems. Dynamic stratigraphy in modern and ancient shallow-marine sequences. Lecture Notes in Earth Sciences, Springer, Berlin, 3, 174 p.

Aigner, T., J. Heinz, J. Hornung and U. Asprion 1998. A hierarchical process-approach to reservoir heterogeneity: examples from outcrop analogues. Bulletin du Centre de Recherches Elf Exploration Production, 22, 1, 1-11.

Kerans, C. and S.W. Tinker 1997. Sequence stratigraphy and characterization of carbonate reservoirs. SEPM Short Course Notes, no. 40, 130 p.

Koehrer, B., M. Zeller, T. Aigner, M. Poeppelreiter, P. Milroy, H. Forke and S. Al-Kindi 2010. Facies and stratigraphic framework of a Khuff outcrop equivalent: Saiq and Mahil formations, Al Jabal al-Akhdar, Sultanate of Oman. GeoArabia, v. 15, no. 2, p. 91-156.

Koehrer, B., T. Aigner and M. Poeppelreiter 2011. Field-scale geometries of Upper Khuff reservoir geobodies in an outcrop analogue (Oman Mountains, Sultanate of Oman). Petroleum Geoscience, v. 17, no. 1, p. 3-16. 
w

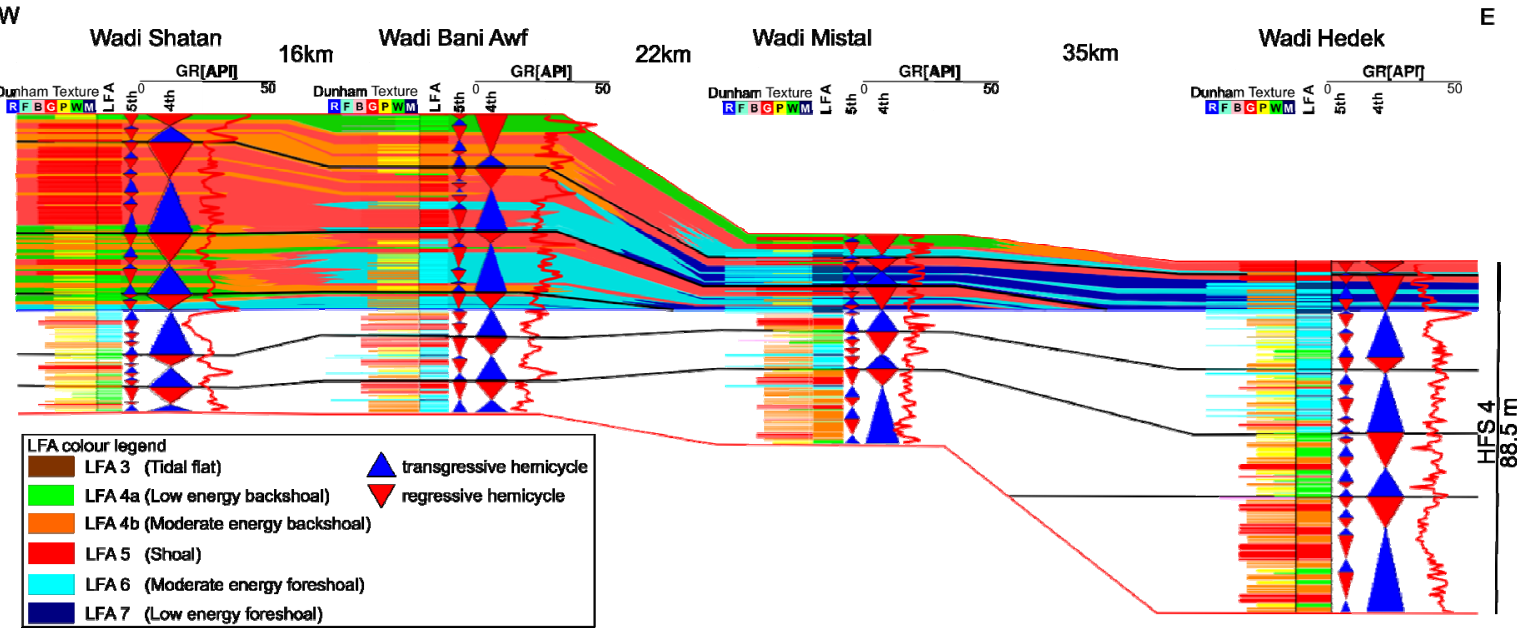

Figure 1: Correlation example of the lowermost high-frequency sequence 4. The maximum flooding of the HFS is used as datum. A clear deepening trend from west to east can be seen in the regressive phase.

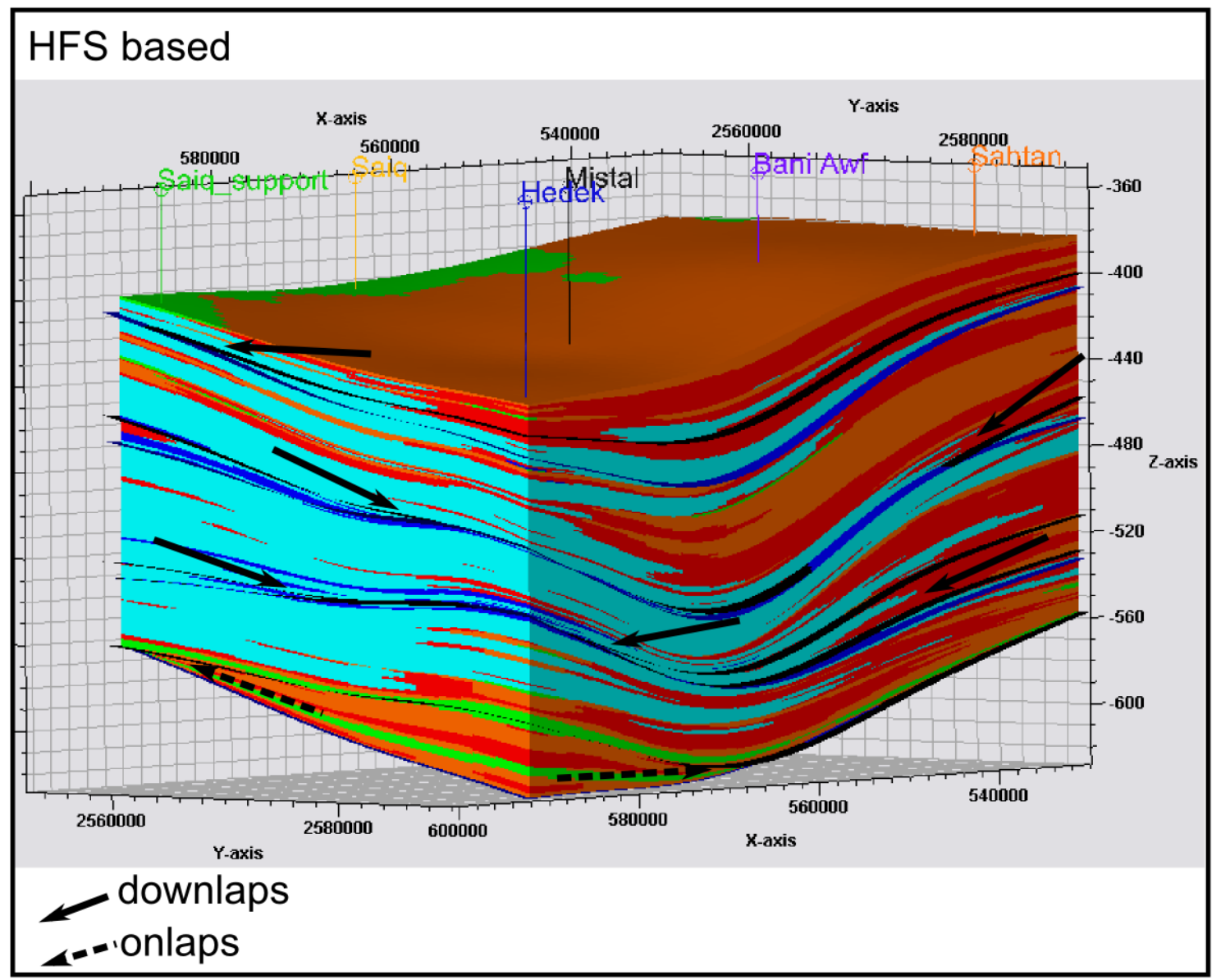

Figure 2: Example of a 3-D model run with the SIS algorithm. It shows the lower part of the smoothed model resulting from 12 realizations. This realization is based on the HFS correlation. Important horizons are displayed to point out the downlapping or onlapping geometries of cycle sets (indicated by the arrows). The colour code is the same as in Figure 1. View is from the northeast. 